\title{
A NOTE ON THE ROYDEN BOUNDARY
}

\author{
BY MOSES GLASNER AND RICHARD KATZ ${ }^{1}$
}

Communicated by Frederick W. Gehring, March 21, 1969

Recently Loeb and Walsh [3] established several results concerning the Royden boundary in the axiomatic setting. This in effect generalizes theorems about HD-functions, Dirichlet-finite harmonic functions on surfaces to Riemannian manifolds, which are the most general carriers of these functions. Their results are however restricted to bounded HD-functions. Whether Nakai's [4] characterization of the HD-functions in terms of the Royden boundary is valid for Riemannian manifolds remains open. The purpose of this note is to announce that Nakai's theory does carry over in its entirety.

The results of Loeb and Walsh include the bounded energy finite solutions of the equation $\Delta u=P u$ with $\int P<\infty$. The boundary that is relevant for these solutions in the axiomatic theory depends heavily on the choice of $P$. The results given below show that the harmonic part of the Royden boundary serves to determine all energy-finite solutions for any $P \geqq 0$.

We now present the precise formulation of our results. Their complete proofs and a more detailed discussion of their relation to the literature will appear in [1], [2].

Let $R$ be a noncompact orientable Riemannian $n$-manifold. Consider the vector lattice $\tilde{M}$ of real-valued functions $f$ on $R$ which have weak exterior derivatives $d f$ with $D(f)=\int_{R} d f \wedge^{*} d f<\infty$. The bounded functions in $\tilde{M}$ form the Royden algebra $M$ associated with $R$. The Royden compactification $R^{*}$ of $R$ is the compact Hausdorff space containing $R$ as an open dense subset such that $M$ extends continuously to $R^{*}$ and also separates points of $R^{*}$.

We say that a sequence $\left\{f_{n}\right\}$ converges to $f$ in the BD-topology on $M$ if it is uniformly bounded, converges to $f$ uniformly on compact subsets of $R$ and $D\left(f-f_{n}\right) \rightarrow 0$. It is known that $M$ is complete. The BD-closure of the functions in $M$ with compact support is denoted by $M_{\Delta}$. The compact set $\Delta=\left\{p \in R^{*} \mid f(p)=0\right.$ for every $\left.f \in M_{\Delta}\right\}$ is known as the harmonic part of the Royden boundary. Among the points of $R^{*}-R$ it is those of $\Delta$ that are significant.

1 The authors are grateful to Professors L. Sario and M. Nakai for making available the manuscript of their forthcoming book Classification theory. Professor John S. M. Chang has independently obtained some of these results in the case $P \equiv 0$. 
Let $P$ be a nonnegative smooth $n$-form on $R$ and denote by $P(R)$ the set of solutions of $d^{*} d u=P u$ on $R$. For $f \in \tilde{M}$, the energy-integral is $E(f)=D(f)+\int_{R} f^{2} P$ and if $g \in \tilde{M}$ set $E(f, g)=\int_{R} d f \wedge^{*} d g+f g P$. Define

$\tilde{E}=\{f \in \tilde{M} \mid E(f)<\infty\}, \quad P D(R)=P(R) \cap \tilde{M}, \quad P E(R)=P(R) \cap \tilde{E}$.

TheOREм 1. Every $f \in \tilde{E}$ has the decomposition $f=u+g$ with $u \in P E(R)$, $g \in \tilde{E}, g \mid \Delta=0$ and $E(u) \leqq E(f)$.

To present the idea of the proof and avoid numerous details we assume $f \geqq 0$ and $P \not \equiv 0$. Choose an exhaustion $\left\{R_{n}\right\}$ of $R$ by regular regions. Let $u_{n}$ be the continuous function on $R$ which is a solution on $R_{n}$ and equal to $f$ on $R-R_{n}$. By Green's formula $E\left(f-u_{n}, u_{n}\right)=0$ and therefore $0 \leqq E\left(f-u_{n}\right)=E(f)-E\left(u_{n}\right)$. Since the sequence $\left\{u_{n}\right\}$ is positive it has a subsequence again denoted by $\left\{u_{n}\right\}$ which converges uniformly on compact subsets to a function $u$ which is a solution or $\infty$. The assumption $P \not \equiv 0$ excludes the possibility $u=\infty$, by virtue of $\int u_{n}^{2} P \leqq E\left(u_{n}\right) \leqq E(f)<\infty$.

Another application of Green's formula shows that $0 \leqq E\left(u_{n}-u_{n+p}\right)$ $=E\left(u_{n}\right)-E\left(u_{n+p}\right)$. Thus $\lim E\left(u_{n}\right)=e$ exists and Fatou's lemma gives $0 \leqq E\left(u_{n}-u\right) \leqq E\left(u_{n}\right)-e$. We conclude that $u_{n}$ converges to $u$ in the $E$-norm and $u \in P E(R)$. Set $g_{n}=f-u_{n}$ and $g=f-u$. Observe that $g_{n}$ has compact support, $g_{n} /\left(1+g_{n}\right) \in M$ and

$$
\frac{g}{1+g}=\mathrm{BD}-\lim \frac{g_{n}}{1+g_{n}} \text {. }
$$

Thus $g /(1+g) \in M_{\Delta}$ which implies that $g \mid \Delta=0$ and completes the proof.

THEOREM 2. For any $u \in P D(R),|u| \leqq \max _{\Delta}|u|$ and if in addition $\min _{\Delta} u \geqq 0$, then $u \geqq 0$.

This shows that the decompositlon in Theorem 1 is unique.

Let $\Delta^{P}$ be the points of $\Delta$ which have neighborhoods $U$ in $R^{*}$ such that $\int_{U \cap R} P<\infty$. Consider the null class $0_{P E}^{m}$ of pairs $(R, P)$ of Riemannian manifolds $R$ and $n$-forms $P$ such that $\operatorname{dim} P E(R) \leqq m$. If $P \equiv 0$, then we make the convention that $0_{P E}^{0}=0_{G}$, the class of parabolic Riemannian manifolds. With this we can state the following consequence of Theorems 1 and 2 .

THEOREM 3. $\Delta^{P}$ consists of exactly $m$ points if and only if $(R, P)$ $\in 0_{P E}^{m}-0_{P E}^{m-1}$.

This in turn gives the following 
Theorem 4. If $P \not \equiv 0$ and $\int_{R} P<\infty$, then $0_{G}=0_{P E}^{0}$.

The harmonic bounded functions are also governed by their behavior near $\Delta$.

THEOREM 5. Let $G$ be a subregion in $R$ and $u$ a bounded harmonic function on $G$. If lim sup $\operatorname{six\rightarrow p}_{\ni \rightarrow p} u(x) \leqq m$ for any $p \in \partial G \cup(\Delta \cap \bar{G})$, then $u \leqq m$.

\section{REFERENCES}

1. M. Glasner and R. Katz, The Royden boundary of a Riemannian manifold, Illinois J. Math (to appear).

2. - On the behavior of $\Delta u=P u$ at the Rouden boundary, J. Analyse Math. (to appear).

3. P. A. Loeb and B. Walsh, An axiomatic approach to the boundary theories of Wiener and Royden, Bull. Amer. Math. Soc. 74 (1968) 1004-1007.

4. M. Nakai, $A$ measure on the harmonic boundary of a Riemann surface, Nagoya Math. J. 17 (1960), 181-218.

California Institute of Technology, Pasadena, California 91109 and

California State College, los Angeles, California 90032 